\title{
Prediction of strain values in reinforcements and concrete of a RC frame using neural networks
}

\author{
Mohammadreza Vafaei ${ }^{1} \cdot$ Sophia C. Alih ${ }^{2} \cdot$ Hossein Shad ${ }^{3} \cdot$ Ali Falah $^{4} \cdot$ Nur Hajarul Falahi Abdul Halim $^{4}$
}

Received: 26 June 2017 / Accepted: 1 January 2018 / Published online: 19 January 2018

(c) The Author(s) 2018. This article is an open access publication

\begin{abstract}
The level of strain in structural elements is an important indicator for the presence of damage and its intensity. Considering this fact, often structural health monitoring systems employ strain gauges to measure strains in critical elements. However, because of their sensitivity to the magnetic fields, inadequate long-term durability especially in harsh environments, difficulties in installation on existing structures, and maintenance cost, installation of strain gauges is not always possible for all structural components. Therefore, a reliable method that can accurately estimate strain values in critical structural elements is necessary for damage identification. In this study, a full-scale test was conducted on a planar RC frame to investigate the capability of neural networks for predicting the strain values. Two neural networks each of which having a single hidden layer was trained to relate the measured rotations and vertical displacements of the frame to the strain values measured at different locations of the frame. Results of trained neural networks indicated that they accurately estimated the strain values both in reinforcements and concrete. In addition, the trained neural networks were capable of predicting strains for the unseen input data set.
\end{abstract}

Keywords Damage detection $\cdot$ Neural networks $\cdot$ Strain measurement $\cdot \mathrm{RC}$ frame $\cdot$ Concrete structure

\section{Introduction}

Sudden collapses of bridges worldwide have increased the attention of researchers to the integrity assessment of in-service structures. While visual inspections and non-destructive tests have been widely employed for the safety assessment of structures, because of their shortcomings, many advanced damage identification methods have been developed in the recent decades (Shahsavari et al. 2017; Janeliukstis et al. 2017). In general, these damage identification methods

Mohammadreza Vafaei

vafaei@utm.my

1 Center for Forensic Engineering, Faculty of Civil Engineering, Universiti Teknologi Malaysia, Johor Bahru, Johor, Malaysia

2 Institute of Noise and Vibration, Faculty of Civil Engineering, Universiti Teknologi Malaysia, Johor Bahru, Johor, Malaysia

3 Department of Civil Engineering, Hakim Sabzevari University, Sabzevar, Khorasan Razavi, Iran

4 Faculty of Civil Engineering, Universiti Teknologi Malaysia, 81310 Johor Bahru, Johor, Malaysia can be categorized into the time and frequency domain approaches. While time domain approaches make use of structural responses (e.g., displacements and accelerations), frequency domain methods take advantage of the change in modal parameters (e.g., natural frequencies and mode shapes). Because of their proven capabilities in pattern recognition and feature extraction, artificial neural networks (ANNs) have been extensively employed by researchers both in the time domain (Vafaei et al. 2011, 2013) and the frequency domain (Vafaei et al. 2015; Vafaei and Alih 2017) damage identification methods. When ANNs are trained, they are able to produce reasonable outputs for the unseen inputs during their training. So far, different types of input and output parameters have been used for the training of ANNs (de Lautour and Omenzetter 2009; Vafaei et al. 2014). In this study, ANNs have been utilized to estimate the strain values in reinforcements and concrete of a full-scale RC frame. When strain values are measured at critical locations of structures, they can be directly related to the intensity of imposed damages. Therefore, strain value can be considered as an important parameter for structural health monitoring. The conducted research in this study is an effort for reducing the number of installed strain gauges on structures by 
estimating the value of strains at required locations through ANNs. In addition, this study is a solution for estimating the strain values in locations where installation of strain gauges is not possible or is very difficult and costly.

\section{Experimental test}

\section{Test structure}

As shown in Fig. 1, the test structure is a full-scale one-bay planer RC frame. The beam of the frame has a rectangular cross-section with the dimensions of $0.3 \times 0.2 \mathrm{~m}$. The length of the beam is $3.6 \mathrm{~m}$. Columns are $3.3 \mathrm{~m}$ in length and have a square cross-section with the dimensions of $0.35 \times 0.35 \mathrm{~m}$. The beam is reinforced by two $16 \mathrm{~mm}$ ribbed bars at the top and bottom of its cross-section. Columns are longitudinally reinforced by eight $16 \mathrm{~mm}$ ribbed bars. The transverse reinforcements of the beam consist of $8 \mathrm{~mm}$ ribbed bars installed at the distances of 0.06 and $0.12 \mathrm{~m}$ as shown in Fig. 1. Columns are transversally reinforced with $8 \mathrm{~mm}$ ribbed bars with the distance of $0.15 \mathrm{~m}$ along their length. At the beam-to-column joints, the distance between transverse reinforcements of columns is reduced to $0.07 \mathrm{~m}$. The compressive strength of the concrete used for the construction of the frame is $30 \mathrm{MPa}$. The yield stress of reinforcements is $570 \mathrm{MPa}$. As shown in Fig. 2, the frame is loaded gradually by a hydraulic jack using a 2-point loading configuration. The load is applied to the beam gradually and its intensity at each step is measured by a load cell. The beam-to-column joint rotations are measured at each loading step for both end corners of the beam. The vertical displacements of the beam are recorded via two LVDTs located at mid-span and 1/3span. As can be seen from Fig. 2, strain values on the surface of concrete are measured at three locations. The first strain gauge (see SC1 in Fig. 2) was installed at the top side of the left corner of the beam, $0.1 \mathrm{~m}$ away from the column face. The other two strain gauges were installed at the bottom side of the beam at mid-span (see SC2 in Fig. 2) and 1/3-span (see SC3 Fig. 2). In addition to the strain values in concrete, strains are also measured at three locations along the longitudinal reinforcements of the beam. Two strain gauges that are referred to as SR 1 and SR2 measure the strain values of top reinforcements of the beam, respectively, $0.1 \mathrm{~m}$ away from the left and right columns' face. The third strain gauge that is referred to as SR3 measures the strain values of reinforcements at the bottom of the beam at mid-span.

\section{Experimental test results}

The frame was loaded gradually in 110 steps until the beam reached to its ultimate capacity. At each loading step, the values of applied load, vertical displacements, and the beam's end corner rotations were recorded. Figure 3a, b

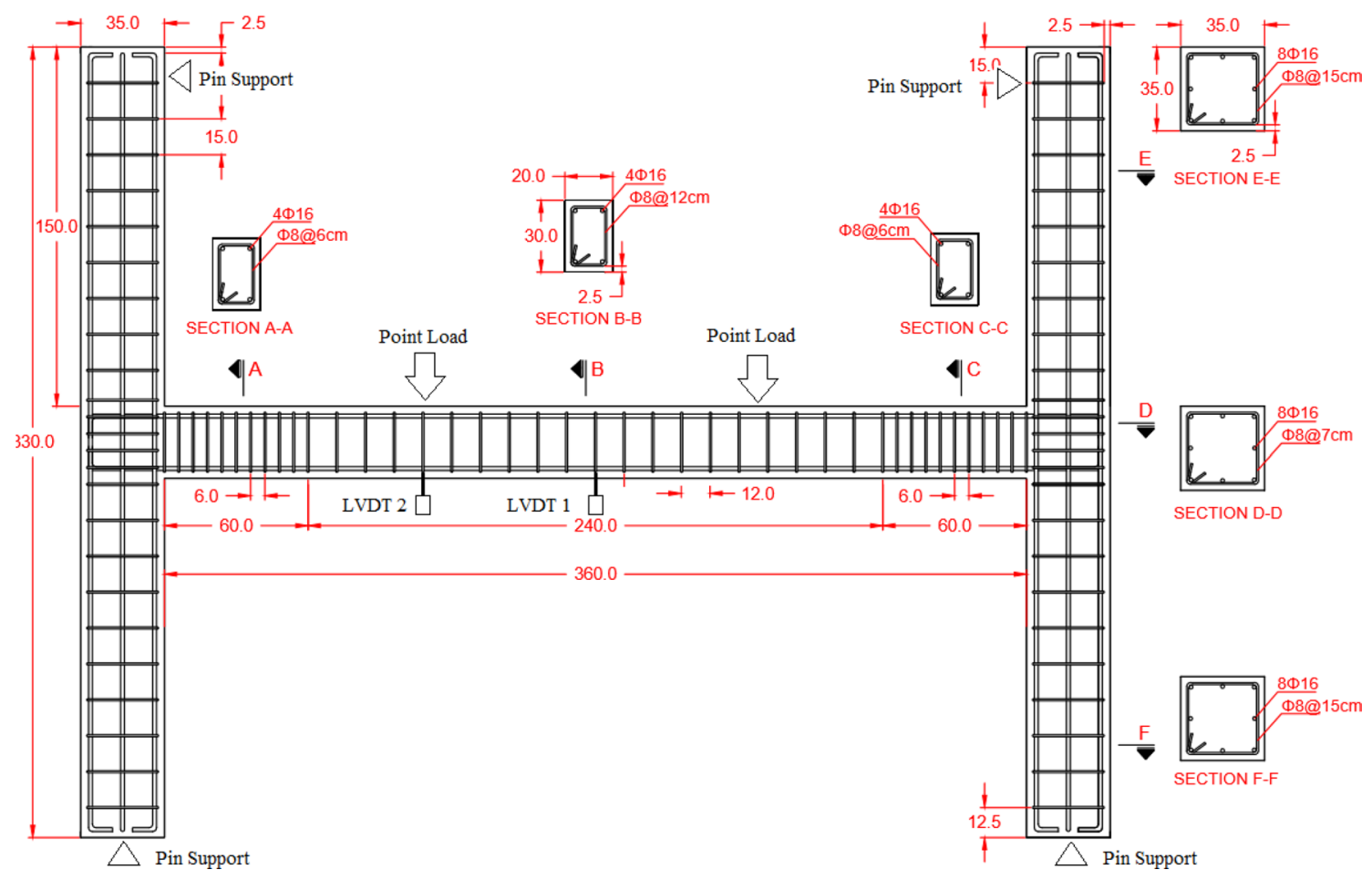

Fig. 1 Details of RC frame tested in this study 
Fig. 2 Test set-up and instrumentation

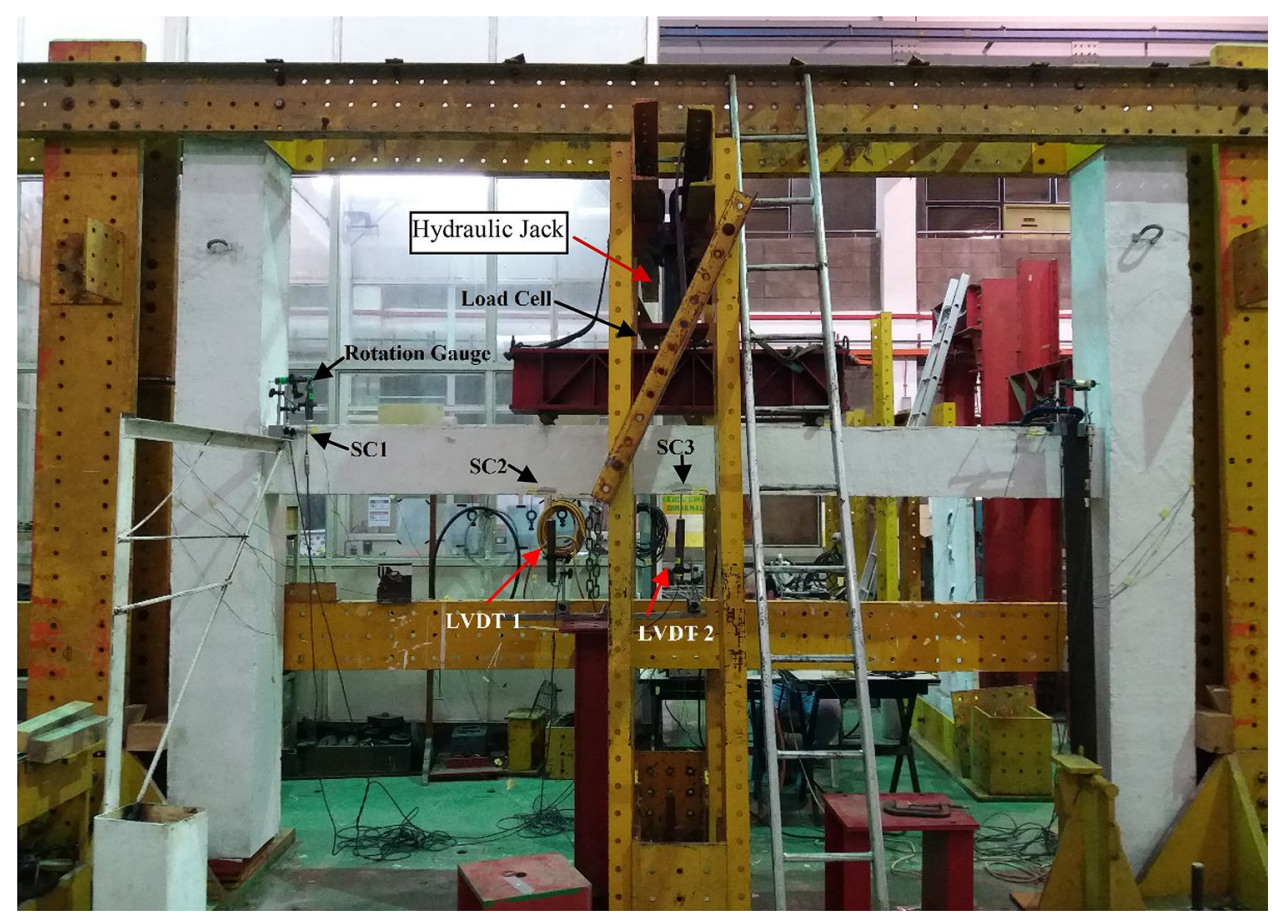

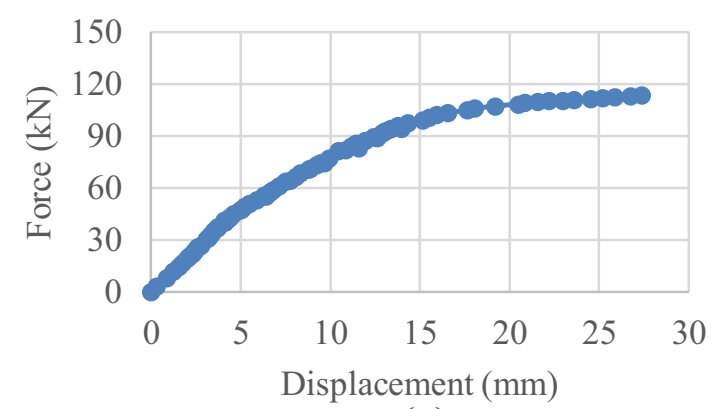

(a)

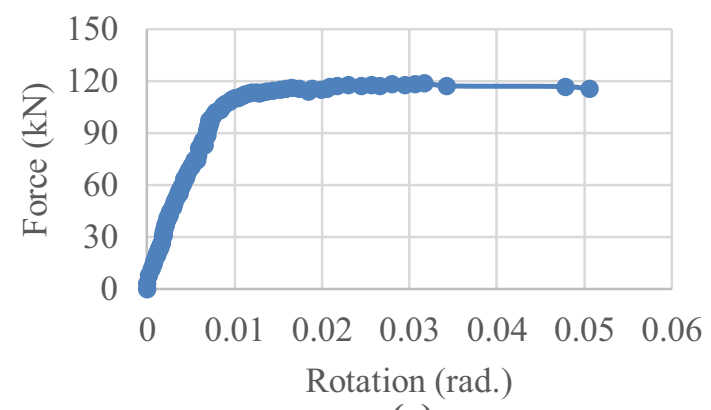

(c)

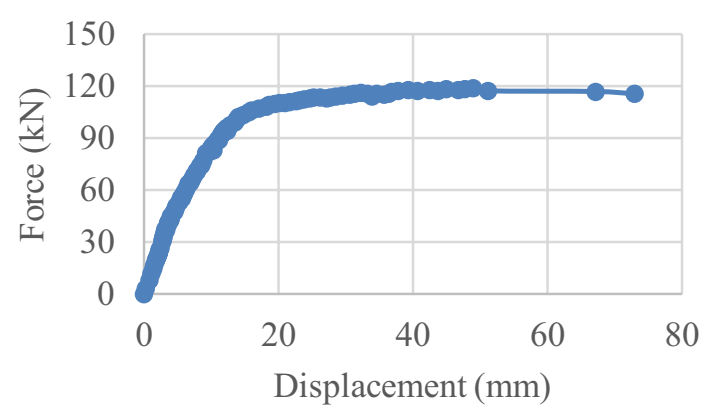

(b)

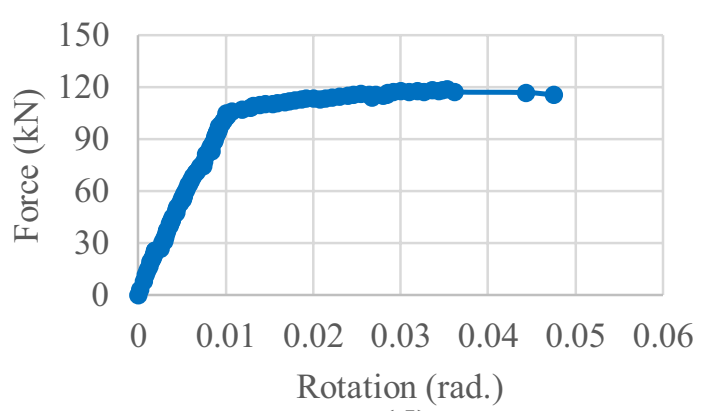

(d)

Fig. 3 Force-displacement and force-rotation relationships. a 1/3-span, $\mathbf{b}$ mid-span, $\mathbf{c}$ rotation at the left end corner of the beam, $\mathbf{d}$ rotation at the right end corner of the beam

display the obtained relationships between the applied load and the vertical displacements measured at the mid-span and 1/3-span, respectively. As can be seen from these figures, the ultimate load capacity of the beam is $119 \mathrm{kN}$.
Figure $3 \mathrm{~b}$ shows that the beam reaches its yield strength when the applied load is around $106 \mathrm{kN}$ and its corresponding displacement at mid-span is $16 \mathrm{~mm}$. Figures 3c, $\mathrm{d}$ display the relationships between the applied load and 
the obtained rotations (i.e., rotation of beam plus rotation of column) for the left and right end corners of the beam, respectively. These figures show that at the ultimate load of the beam, the rotations at the left and right end corners are 0.036 and 0.031 radians, respectively. Moreover, at the yield strength, rotations at the left and right end corners of the beam reach to 0.008 and 0.011 radians, respectively. Figure 4 shows the obtained relationships between the applied load and strain values of the concrete. It can be seen that the measured strains at the mid-span show higher values compared to those measured at the 1/3-span. This can be also seen that the measured strains at the top left end corner of the beam (i.e., SC1) are significantly lower than those measured at the mid-span and 1/3-span. The graphs shown in Fig. 4 indicate that the relationships between the applied forces and the measured strains on the surface of the concrete are highly nonlinear. As shown in Fig. 5, similar nonlinear relationships can be observed between the applied forces and the measured strains for reinforcements. Figure 5 shows that strain values measured at the top left end corner of the beam are larger than those measured at the right end corner which correlates with the obtained rotations for the both end corners. It is noteworthy that strain values measured for the reinforcements at the mid-span are smaller than those measured at the both end corners.

\section{Estimation of strain values}

\section{Neural network design}

As Figs. 4 and 5 showed, there were highly nonlinear relationships between the applied load and the measured strain values. Neural networks have demonstrated their capability in dealing with such nonlinear relationships. In this study, two neural networks with a similar architecture were designed to examine their capability in estimating the values of strains in the concrete and reinforcements. As shown in Fig. 6, the employed neural network is a supervised feed-forward multi-layer neural network with a fully connected configuration. The number of hidden layers and their neurons depend on the complexity of problems and often are determined by trial and errors. It has been shown in many damage identification studies that the usage of one hidden layer is adequate for accurate feature extraction and pattern recognition (Yam et al. 2003; Zang and Imregun 2001). Therefore, as can be seen from Fig. 6, both neural networks made use of one hidden layer. The numbers of neurons in the hidden layer were determined through trial and errors considering the performance of the neural networks which was calculated by the mean squared error (MSE). It should be noted that there are some rules of the thumb to estimate the number of neurons

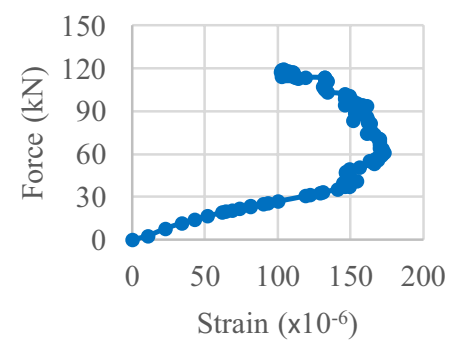

(a)

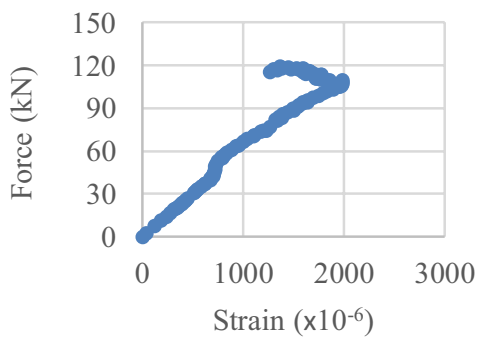

(b)

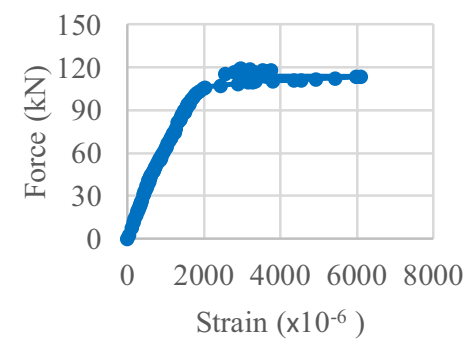

(c)

Fig. 4 Measured strain values on the surface of concrete. a Top left, SC1. b Bottom 1/3-span, SC2. c Bottom, mid-span, SC3

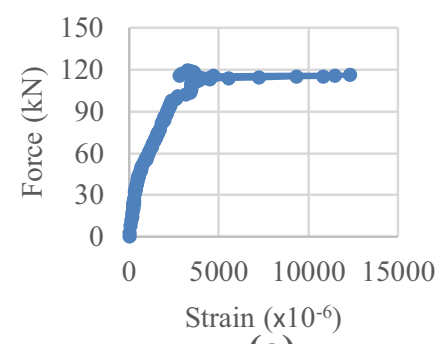

(a)

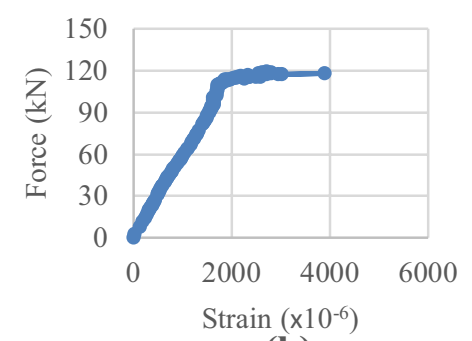

(b)

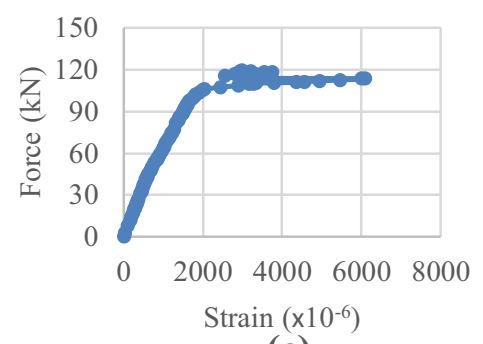

(c)

Fig. 5 Measured strain values for the longitudinal reinforcements of the beam. a Top left end corner, SR1. b Top right end corner, SR2. c Bottom mid-span, SR3 


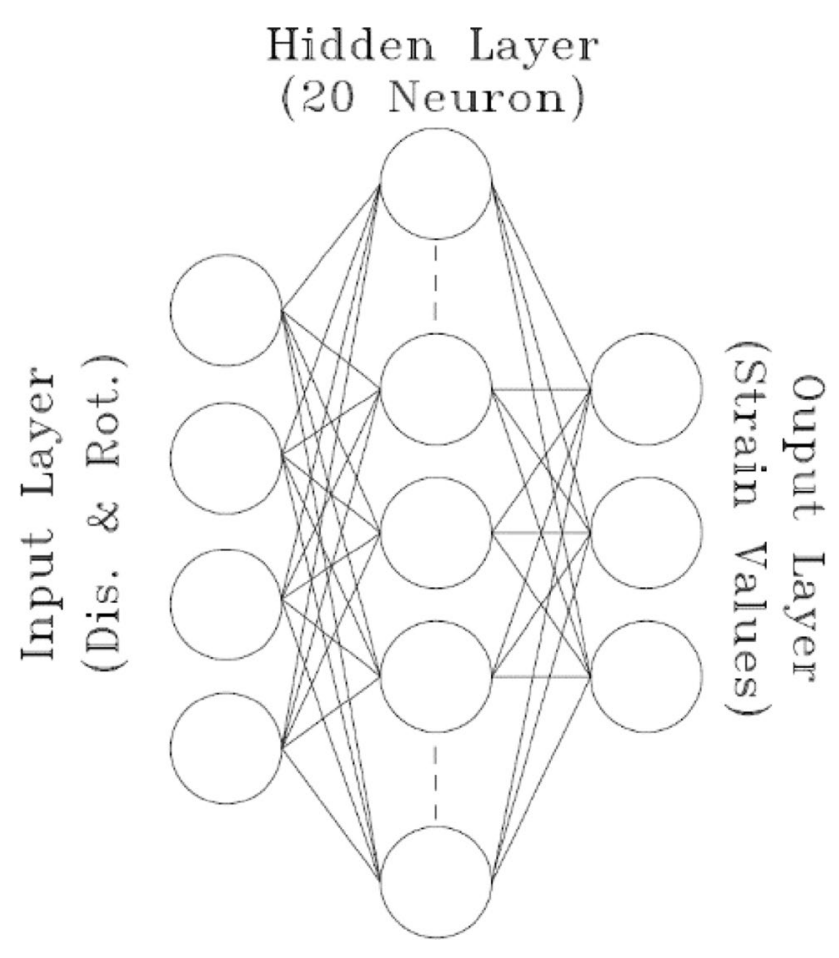

Fig. 6 Architecture of the employed neural network

Table 1 Performance of trained neural networks considering different neurons for the hidden layer

\begin{tabular}{llllll}
\hline No. of neurons & 10 & 15 & 20 & 25 & 30 \\
\hline MSE of learning & 0.012 & 0.008 & 0.004 & 0.007 & 0.009 \\
MSE of testing & 0.010 & 0.012 & 0.007 & 0.009 & 0.007 \\
MSE of validation & 0.023 & 0.019 & 0.009 & 0.012 & 0.0011 \\
\hline
\end{tabular}

inside the hidden layer of a neural networks like the one proposed by Kermanshahi (1999). However, the rule of thumb has no theoretical background and may not be generally applicable (Parhi and Dash 2011). Therefore, similar to other researchers (Xu et al. 2004; Pandey and Barai 1995), herein, the trial and error method was adopted. As can be seen from Table 1, the best performance was achieved when the hidden layer had 20 neurons. It should be mentioned that, Table 1 displays the obtained results for the first neural network; however, similar results were obtained for the second network. The input layer of the both networks had 4 neurons and included the measured vertical displacements at mid-span and 1/3-span together with the rotations of the beam at its both end corners. The output layer of the neural networks had 3 neurons. For the first network, strain values measured at the three locations of concrete were considered as the output vector. However, for the second network, the measured strains of reinforcements at the three locations were considered as the output layer. Totally, 110 data sets were obtained from experimental tests. The early stopping method was used for training of neural networks. According to this method, data should be divided into three groups of training, testing and validation. Herein, $70 \%$ of data was randomly allocated to training and the rest were equally divided between validation and testing data. In addition, out of 110 data, 10 data sets were selected from the linear and nonlinear part of the load-displacement relationships as unseen data. The unseen data set were not included in the training of neural networks. They were employed to check on the generalization capability of the trained neural networks and unbiased split of data for linear and nonlinear part of load-displacement relationships (Reitermanova 2010).

The Levenberg-Marquardt (LM) backpropagation algorithm was employed for training the neural networks. Moreover, the Gradient descent weight/bias learning function was used. The hyperbolic tangent function was also used as the activation function of both neural networks. To avoid the saturation of neural networks, the input and output data were scaled to $[-1,1]$. Moreover, $70 \%$ of data were allocated to the training and $30 \%$ were equally assigned to testing and validation. The performance of the neural networks was monitored by validation samples to avoid over-fitting. Testing samples were used to check on the generalization ability of the trained neural networks.

\section{Results of the trained neural networks}

The obtained results from the trained neural networks are presented in Figs. 7 and 8. As can be seen from correlation coefficients $\left(R^{2}\right)$ shown in Fig. 7, the first neural network has accurately estimated the values of strains on the surface of concrete for the three measured locations. These results imply that the trained neural network not only has been able to predict the strain values accurately for the linear range of the beam's force-displacement relationship, but it has also successfully predicted the strain values for the nonlinear range. The maximum prediction errors for SC1, SC2 and SC3 are, respectively, 11.1, 12.7 and 27.5\%. The correlation coefficients obtained for Fig. 8 indicate that the second neural network has also accurately predicted the strain values in reinforcements for all the three studied locations. The maximum prediction errors for SR1, SR2 and SR3 are, respectively, 12.1, 28.7 and 12.4\%. Figure 9 displays the obtained results for the unseen data set. It is evident from this figure that the trained neural networks have successfully predicted the strain values on concrete and reinforcements demonstrating the generalization capability of the trained neural networks. In short, this can be concluded that ANNs are potential tools for estimation of strains in structures. 


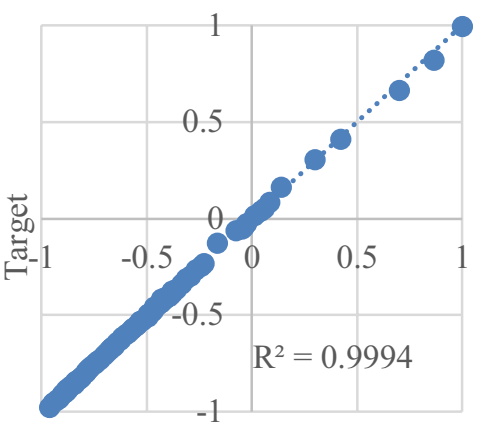

Prediction

(a)

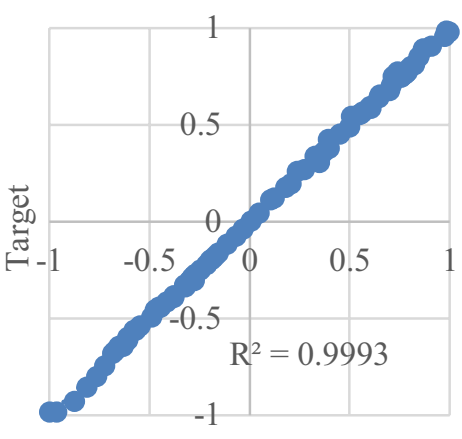

Prediction

(b)

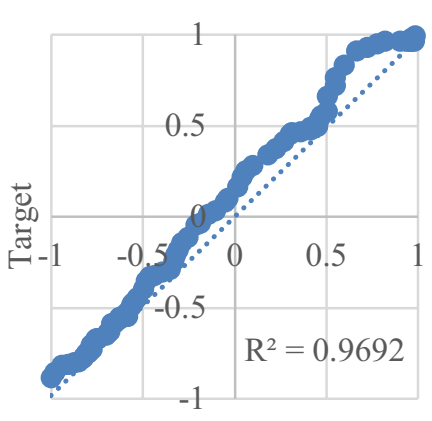

Prediction

(c)

Fig. 7 Comparison between predictions of the neural network and the targets for strain values obtained for concrete surface. a SC1, b SC2, c SC3

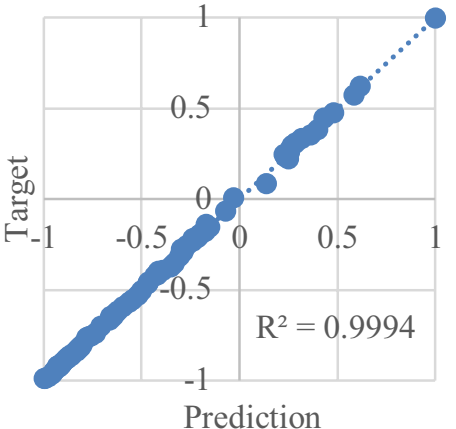

(a)

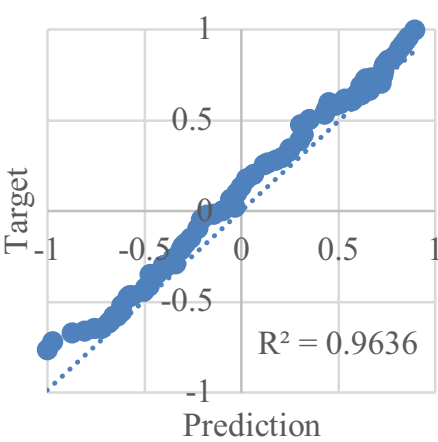

(b)

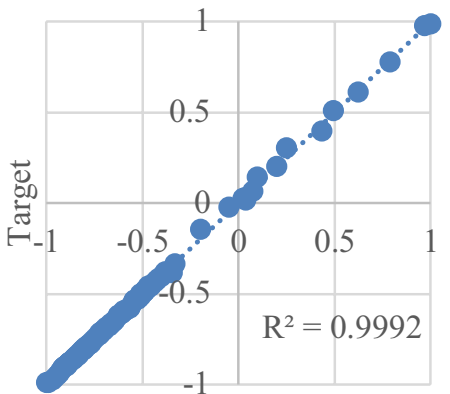

Prediction

(c)

Fig. 8 Comparison between predictions of the neural network and the targets for strain values obtained for reinforcements. a SR1, b SR2, c SR3

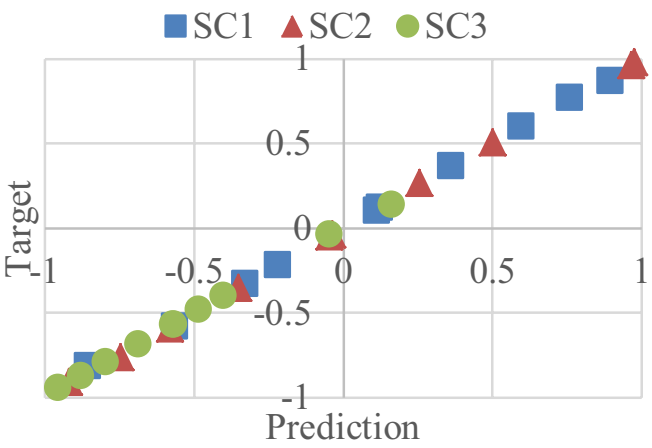

(a)

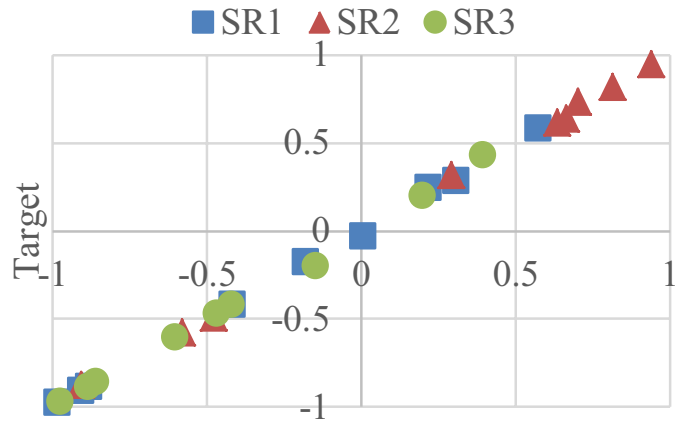

Prediction

(b)

Fig. 9 Comparison between predictions of the neural networks and the targets for unseen data set. a Measured strains on the surface of concrete, b measured strains in reinforcements 


\section{Conclusions}

In this study, artificial neural networks were used to estimate the value of strains on the surface of concrete and in reinforcements. A full-scale RC frame was constructed and loaded gradually until reaching to its ultimate capacity. The vertical displacements were measured at $1 / 3$-span and midspan of the beam of the frame. Moreover, the rotations of the end corners of the beam were measured at each loading step. The values of strains were also measured at three different locations for concrete and reinforcements. From experimental tests, totally 110 data sets were obtained. Two supervised feed-forward multi-layer neural networks were designed. The neural networks had one hidden layer with 20 neurons. The input layer of the neural networks had four nodes, while the output layer had three nodes. Vertical displacements and end corner rotations were considered as the input parameters to the neural networks, while the strain values in the selected locations were considered as the output parameters. The neural networks were trained using 100 data set obtained from the experimental tests. Results indicated that the values of strains at all selected locations were accurately estimated by the trained neural networks both for concrete and reinforcements. In addition, the trained neural networks predicted the value of strains accurately for 10 unseen data sets which were not included in their training procedure.

Acknowledgements The authors are thankful to the comments from anonymous reviewers. In addition, the financial support from Malaysian Ministry of Higher Education under the Grant No. R.J130000.7822.4F760 is greatly acknowledged.

Open Access This article is distributed under the terms of the Creative Commons Attribution 4.0 International License (http://creativecomm ons.org/licenses/by/4.0/), which permits unrestricted use, distribution, and reproduction in any medium, provided you give appropriate credit to the original author(s) and the source, provide a link to the Creative Commons license, and indicate if changes were made.

\section{References}

de Lautour OR, Omenzetter P (2009) Prediction of seismic-induced structural damage using artificial neural networks. Eng Struct 31(2):600-606
Janeliukstis R, Rucevskis S, Wesolowski M, Chate A (2017) Experimental structural damage localization in beam structure using spatial continuous wavelet transform and mode shape curvature methods. Measurement 102:253-270

Kermanshahi B (1999) Design and application of neural networks (chapter 3). Shokodo, Tokyo

Pandey PC, Barai SV (1995) Multilayer perceptron in damage detection of bridge structures. Comput Struct 54(4):597-608

Parhi DR, Dash AK (2011) Application of neural network and finite element for condition monitoring of structures. Proc Inst Mech Eng Part C J Mech Eng Sci 225(6):1329-1339

Reitermanova Z (2010) Data splitting. In: 19th annual conference of doctoral students WDS'10. Part I-mathematics and computer sciences, vol 10, pp 31-36. Prague, Czech Republic

Shahsavari V, Chouinard L, Bastien J (2017) Wavelet-based analysis of mode shapes for statistical detection and localization of damage in beams using likelihood ratio test. Eng Struct 132:494-507

Vafaei M, Alih SC (2017) Adequacy of first mode shape differences for damage identification of cantilever structures using neural networks. Neural Comput Appl. https://doi.org/10.1007/s005 21-017-2846-6

Vafaei M, bin Adnan A, Yadollahi M. (2011) Seismic damage detection using pushover analysis. In: Advanced materials research, vol 255, pp 2496-2499. Trans Tech Publications. doi:https://doi. org/10.4028/www.scientific.net/AMR.255-260.2496

Vafaei M, Adnan AB, Abd. Rahman AB (2013) Real-time seismic damage detection of concrete shear walls using artificial neural networks. J Earthq Eng 17(1):137-154

Vafaei M, Adnan AB, Abd. Rahman AB (2014) A neuro-wavelet technique for seismic damage identification of cantilever structures. Struct Infrastruct Eng 10(12):1666-1684

Vafaei M, Alih SC, Rahman ABA, Adnan AB (2015) A wavelet-based technique for damage quantification via mode shape decomposition. Struct Infrastruct Eng 11(7):869-883

Xu B, Wu Z, Chen G, Yokoyama K (2004) Direct identification of structural parameters from dynamic responses with neural networks. Eng Appl Artif Intell 17(8):931-943

Yam LH, Yan YJ, Jiang JS (2003) Vibration-based damage detection for composite structures using wavelet transform and neural network identification. Compos Struct 60(4):403-412

Zang C, Imregun M (2001) Structural damage detection using artificial neural networks and measured FRF data reduced via principal component projection. J Sound Vib 242(5):813-827

Publisher's Note Springer Nature remains neutral with regard to urisdictional claims in published maps and institutional affiliations. 